\title{
Spontaneous Action Potentials and Neural Coding in Unmyelinated Axons
}

\section{Cian O'Donnell}

cian@salk.edu

Institute for Adaptive and Neural Computation, School of Informatics, University

of Edinburgh, Edinburgh EH8 9AB, U.K., and Computational Neurobiology

Laboratory, Salk Institute for Biological Studies, La Jolla, CA, U.S.A.

\section{Mark C. W. van Rossum}

mvanross@inf.ed.ac.uk

Institute for Adaptive and Neural Computation, School of Informatics,

University of Edinburgh, Edinburgh EH8 9AB, U.K.

The voltage-gated $\mathrm{Na}$ and $\mathrm{K}$ channels in neurons are responsible for action potential generation. Because ion channels open and close in a stochastic fashion, spontaneous (ectopic) action potentials can result even in the absence of stimulation. While spontaneous action potentials have been studied in detail in single-compartment models, studies on spatially extended processes have been limited. The simulations and analysis presented here show that spontaneous rate in unmyelinated axon depends nonmonotonically on the length of the axon, that the spontaneous activity has sub-Poisson statistics, and that neural coding can be hampered by the spontaneous spikes by reducing the probability of transmitting the first spike in a train.

\section{Introduction}

Action potentials rely crucially on the voltage-gated channels in the neural membrane. The classic Hodgkin-Huxley model describes how small depolarizations of the membrane lead to increased opening of the Na channels, the ion flux through which further depolarizes the membrane. Once this process reaches a point of no return, an action potential is generated, which is terminated by the opening of the hyperpolarizing $\mathrm{K}$ channels that follow the membrane potential more sluggishly than the Na channels. As the opening of the channels is a stochastic process, the action potentials have a stochastic character as well. This is most noticeable in the initiation phase

C.O'D. is currently at Computational Neurobiology Laboratory, Salk Institute for Biological Studies, La Jolla, CA, U.S.A. 
of the spike when only a few channels are open. During the later phase, so many channels open that stochastic fluctuations are largely averaged out.

The stochastic character of ion channels has been studied extensively in both the experimental and modeling literature. A number of modeling studies have considered single-compartment models, in which a cell is lumped together in one isopotential sphere. For spheres with small membrane areas, stochastic opening and closing of channels can lead to spontaneous action potentials, even in the absence of any stimulation. For large membrane areas, the stochastic character disappears and the system is well described by the deterministic Hodgkin-Huxley equations. In particular, the rate of spontaneous action potentials decreases exponentially with the membrane area (see below and Chow \& White, 1996).

Similarly, for unmyelinated axons, the spontaneous action potential rate increases as the diameter of the axon thins. It has been argued using simulations that in $1 \mathrm{~mm}$ long axons thinner than about $0.1 \mu \mathrm{m}$, spontaneous firing rates become so substantial that they severely interfere with the communication of stimulus-driven action potentials (Faisal, White, \& Laughlin, 2005). This effect might therefore impose a limit on the minimal diameter of axons, but length dependence has not been examined.

In this study we examine in simulations the relation of the spontaneous rate and the axon length. Interestingly, depending on diameter, the spontaneous rate is nonmonotonic in length; it is high for both short and long axons and lowest for intermediate-length axons. Our subsequent reduced model allows us to explore these effects exactly and identifies various regimes, each with its particular characteristics. Next, we show how spontaneous spikes interfere with information coding. The spontaneous spikes have peculiar characteristics different from any other sources of noise studied before in neurons; they can hamper coding but can also be used to identify spontaneous spiking activity experimentally.

\section{Results}

We consider a uniform, unmyelinated axon with sealed ends and use the standard Hodgkin-Huxley $(\mathrm{HH})$ model to describe the active properties of the axon. The only difference in the standard parameters is that we vary the diameter and length of the axon. While the $\mathrm{HH}$ model at a temperature of $6.3^{\circ} \mathrm{C}$ can hardly be called a realistic model for mammalian neurons, we use it because the full characterization of ion channels in mammalian unmyelinated axons is incomplete. Furthermore, using the $\mathrm{HH}$ model has the advantage that both its deterministic and stochastic variants have been studied extensively. The stochastic simulation were done using both the PSICS simulator (Cannon, O'Donnell, \& Nolan, 2010) and the stochastic module in NEURON (Carnevale \& Hines, 2006). 

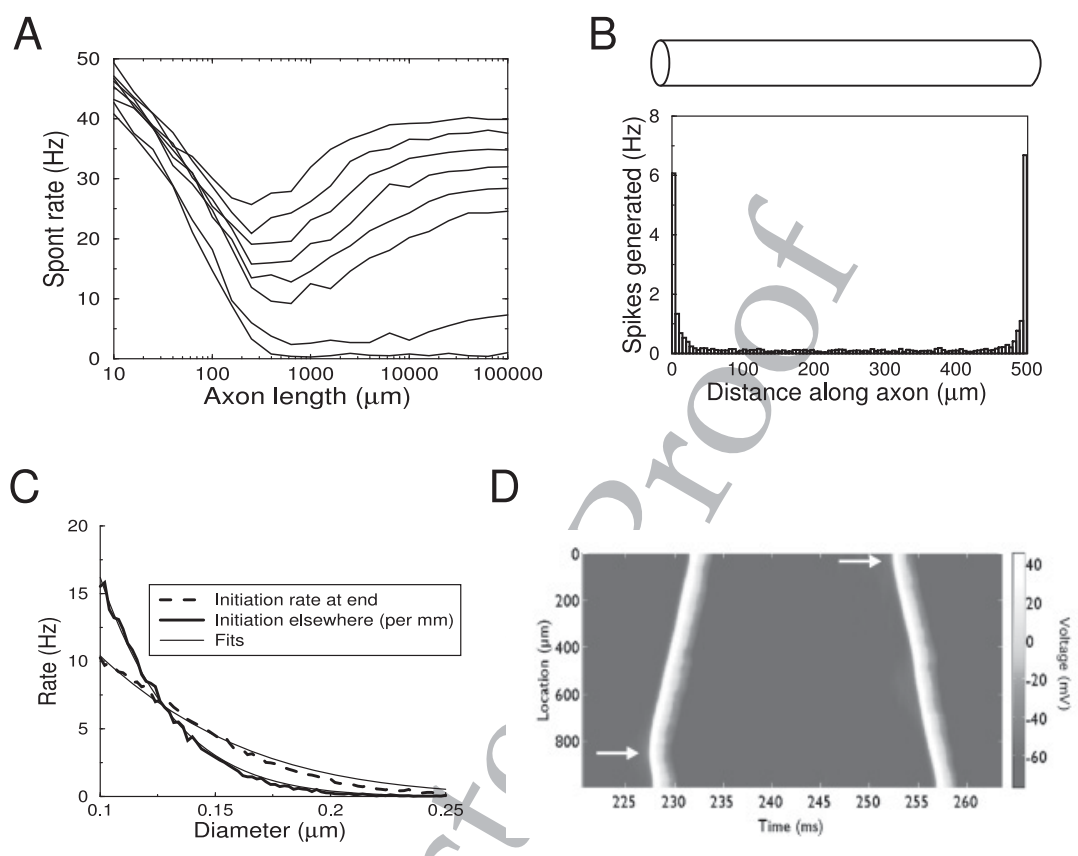

Figure 1: Spontaneous action potentials in Hodgkin-Huxley axons with uniform, fixed channel densities as a function length and width. (A) The total spontaneous action potential rate as a function of axon length for diameters (top to bottom curve): $0.1,0.11,0.12,0.13,0.14,0.15,0.2$, and $0.25 \mu \mathrm{m}$. (B) The spike generation rate along an axon. Compartments at the ends are hot spots that generate many more spikes per compartment than the rest of the axon. Axon of $500 \mu \mathrm{m}$ length and $0.1 \mu \mathrm{m}$ diameter. (C) The spontaneous spike initiation rates at the ends $\left(r_{e}\right.$, in $\left.\mathrm{Hz}\right)$ and in the rest of the axon $\left(\rho_{m}\right.$, in $\mathrm{Hz}$ per mm) versus the diameter. Extracted from a simulation of an axon of $1 \mathrm{~mm}$ length. Thin lines indicate fits to theory (see the text). At the diameter at which the curves intersect, there are as many spikes generated at one end as there are in the rest of this $1 \mathrm{~mm}$ axon. (D) Short segment of a simulation showing the generation of two spontaneous spikes (arrows).

2.1 Rate Versus Length. We first study the spontaneous rate in axons of arbitrary diameter and length, (see Figure 1A). For a given diameter the spontaneous rate is nonmonotonic in the length: it is high for very short and very long axons but lower for intermediate lengths. This behavior can be understood as follows. When the axon is very long, it can be seen as a collection of many weakly coupled compartments, each of which can generate spikes. Yet once a spike is generated, it will travel along the whole axon (unless it collides with another spontaneous spike; see below). As the number of such compartments and thus the number of independent 
initiation zones increases with axon length, the rate increases with length for long enough axons. However, for very long axons, the rate saturates due to collisions.

In contrast, very short axons can be collapsed into a single compartment if they are much shorter than the electrotonic length (or space constant) $\Lambda$. Even in the single-compartment case, the analysis of spontaneous spiking in the HH model is complicated (see Chow \& White, 1996, and corrections in Keener \& Newby, 2011; Newby, Bressloff, \& Keener, 2013; O'Donnell \& Van Rossum, 2014). Fortunately the dependence of the spontaneous rate on area and thus length is well understood (Chow \& White, 1996). In analogy with a Kramer's escape problem, the spontaneous firing rate $f$ behaves as

$$
f \propto \exp \left(-c / \sigma_{V}^{2}\right)
$$

where $\sigma_{V}^{2}$ is variance in the membrane voltage and $c$ is some constant. Under the assumption of small fluctuations, the variance in the voltage equals $\sigma_{V}^{2}=$ $\int S_{I}(f)|Z(f)|^{2} d f$, where $S_{I}(f)$ is the power spectrum of the noise current and $Z(f)$ the membrane impedance. This voltage variance is in general a complicated expression, as the channel noise has a colored power spectrum and the membrane impedance in the $\mathrm{HH}$ model has a resonance $\left(\mathrm{O}^{\prime}\right.$ Donnell \& Van Rossum, 2014). However, here we need only the dependence on the membrane area. Assuming that the voltage is approximately constant, the channels are independent and the spectral density of the fluctuations in the current is proportional to the number of channels and hence proportional to the membrane area $S_{I}(f) \propto A$. However, the impedance is inversely proportional to area, $Z(f) \propto 1 / A$, so we find that $\sigma_{V}^{2} \propto 1 / A$ and $\log f \propto$ $-A \propto-L$. This exponential dependence on length for short axons together with the behavior for long axons determines the shape of Figure 1A: the spontaneous rate increases for both very short and very long axons.

2.2 Effect of Diameter and Spike Initiation Zones. While spikes can be generated anywhere in the axon, Figure 1D, the initiation is not uniform. A histogram of the initiation location shows that most spikes are generated at the ends of the axon (see Figure 1B). This can be understood using an argument similar to the one above explaining the area dependence in single compartments. The variance of the noise currents scales with the number of channels in the local neighborhood. There are twice as many neighboring channels in the middle of the axon as there are at the end. However, the impedance at the end is twice that in the middle of the axon as the current can flow in only one instead of two directions (or, equivalently, the current threshold is lower). Thus, at the axon ends, the variance in the voltage is double that in the middle of the axon (Tuckwell \& Walsh, 1983), making it the preferred initiation site. Because of the exponential dependence of spike rate on voltage variance, equation 2.1, many more spikes will be 
produced at the axon ends than in the middle, despite the noise only being double. Also note that the preferred initiation zone extends only a bit into the axon, much less than the electrotonic length $\Lambda$, which is about $80 \mu \mathrm{m}$ (for a $0.1 \mu \mathrm{m}$ diameter $\mathrm{HH}$ axon at rest, including the contributions of $\mathrm{Na}$ and $\mathrm{K}$ channels). The reason is that the channel noise, which contains high frequencies, is filtered within a much shorter distance than the electrotonic length $\Lambda$, which describes the DC decay in a cable.

The total spontaneous generation rate in an axon of arbitrary length is by definition a sum of the generation rate at the ends and the generation rate anywhere else. We define $r_{e}$ as the generation rate at one end (measured in $\mathrm{Hz}$, taken within $40 \mu \mathrm{m}$ from the end) and $\rho_{m}$ as the generation rate anywhere else (measured in $\mathrm{Hz} / \mathrm{mm}$ ), so that the rate at which spikes are created, $f_{\text {created }}$, can be written as

$$
f_{\text {created }}=2 r_{e}+L \rho_{m} \text {, }
$$

where we assume the axon is long enough $(L \gg \Lambda)$ so that we can ignore the length dependence of $r_{e}$ in the left portion of Figure 1A.

These spike initiation rates, $r_{e}$ and $\rho_{m}$, are compared as a function of diameter $d$ in Figure 1C. It shows that both decrease for wider diameters. The dependence on diameter can be understood as follows. The electrical coupling between neighboring compartments increases for wider axons as the axial conductance is larger. Assuming a fixed channel density, wider axons have more channels and hence more noise per length. As a result a larger-diameter axon collects more noise current; however, the impedance is lower as well, so that the net voltage variance is smaller for wider axons. The effective area equals $A \propto d \Lambda \propto d^{3 / 2}$.

To test this, we simulate a $1 \mathrm{~mm}$ long axon and various diameters and extract the number of spikes generated at the ends and elsewhere. The spike generation rates of the simulations are well fitted to $-\log \left(r_{e}\right) \propto\left(d / d_{0}\right)^{3 / 2}$, while $-\log \left(\rho_{m}\right) \propto 2\left(d / d_{0}\right)^{3 / 2}$, with a fit parameter $d_{0} \approx 0.1 \mu \mathrm{m}$. The factor 2 in the fit of $\rho_{m}$ expresses the increased generation rate at the end of the axon explained above. Due to the exponential dependence of the rate on the fluctuations, the ratio of these generation rates is not constant but shifts in favor of end generation for thicker axons, thus contributing to the change in shape of the curves in Figure 1A as the diameter is varied. We define the critical length $L_{c}$ as the length at which the two rates are comparable, $L_{c}=2 r_{e} / \rho_{m}$. It increases approximately exponentially with the diameter in our simulations, (see Figure $2 \mathrm{~B}$ ). For very wide axons, which are better approximated by a single compartment than an infinitely long cable, we would expect $-\log \rho_{m} \propto d L$. This happens when $d \approx 10 \mu \mathrm{m}$ for a $1 \mathrm{~mm}$ long axon. Also for very narrow axons, this theory will break down because spike rates become so high that refractory effects become important and the escape problem analogy no longer holds. 

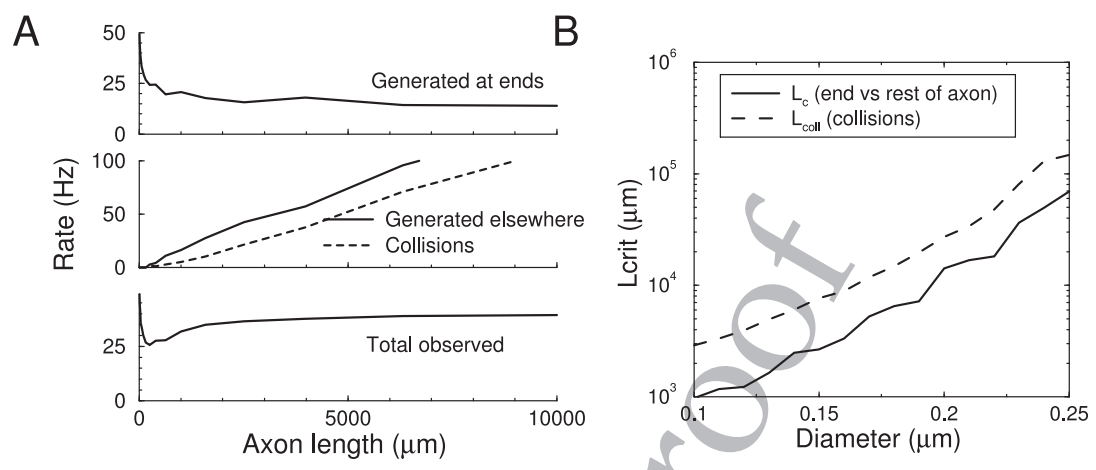

Figure 2: (A) Split of the various contributions to the observed spontaneous rate as a function of axon length (axon with $0.1 \mu \mathrm{m}$ diameter). Top: Rate of spikes generated at the axon ends. Middle: Rate of spikes generated in the rest of the axon and the rate of collisions of spikes in the axon. Bottom: The resulting observed firing rate (the sum of the two generation rates minus the collision rate). (B) The two characteristic length parameters as a function of axon diameter: Solid line $\left(L_{c}\right)$, for axons shorter than this length; most spikes are generated at the ends. For axons longer than this length; most spikes are generated in the rest of the axon. Dashed line $\left(L_{\text {coll }}\right)$ for axons longer than this length; collisions of spikes significantly limit the observed spontaneous rate.

2.3 Collisions. So far we have ignored another important effect for long axons that also contributes to the shape of Figure 1A: two spikes traveling in opposite direction in the axon will collide. When this happens, the spikes annihilate as the membrane on either side of the collision will be in a refractory state and further propagation fails. The probability that spikes collide increases in longer axons. First, the rate increases with length, as we saw in equation 2.2. Second, it takes longer for the spikes to propagate the whole length. The speed of propagation in the $\mathrm{HH}$ model is about $200 \mu \mathrm{m} / \mathrm{ms}$ for a diameter of $0.1 \mu \mathrm{m}$ and increases with diameter as $\sqrt{d}$. Thus, while for short axons, with low rates all initiated spikes travel the full axon, in long axons, the observed spontaneous rate is lower than the initiation rate due to collisions. Note that in the absence of collisions, the observed rate is clearly independent of the observation point, whether at any end of the axon or anywhere in the middle (see Figure 3Ai). Yet it can be observed from diagrams like Figure 3Aii that in the presence of collisions, the observed rate of spikes will be the same at any point of the axon.

With every collision, two spikes merge into one. The observed spontaneous rate, $f_{\text {obs }}$, thus equals the number of spikes generated at the ends, plus the spikes generated anywhere else, minus the collision rate $r_{\text {colls }}$,

$$
f_{\text {obs }}=2 r_{e}+L \rho_{m}-r_{\text {colls }} \text {. }
$$




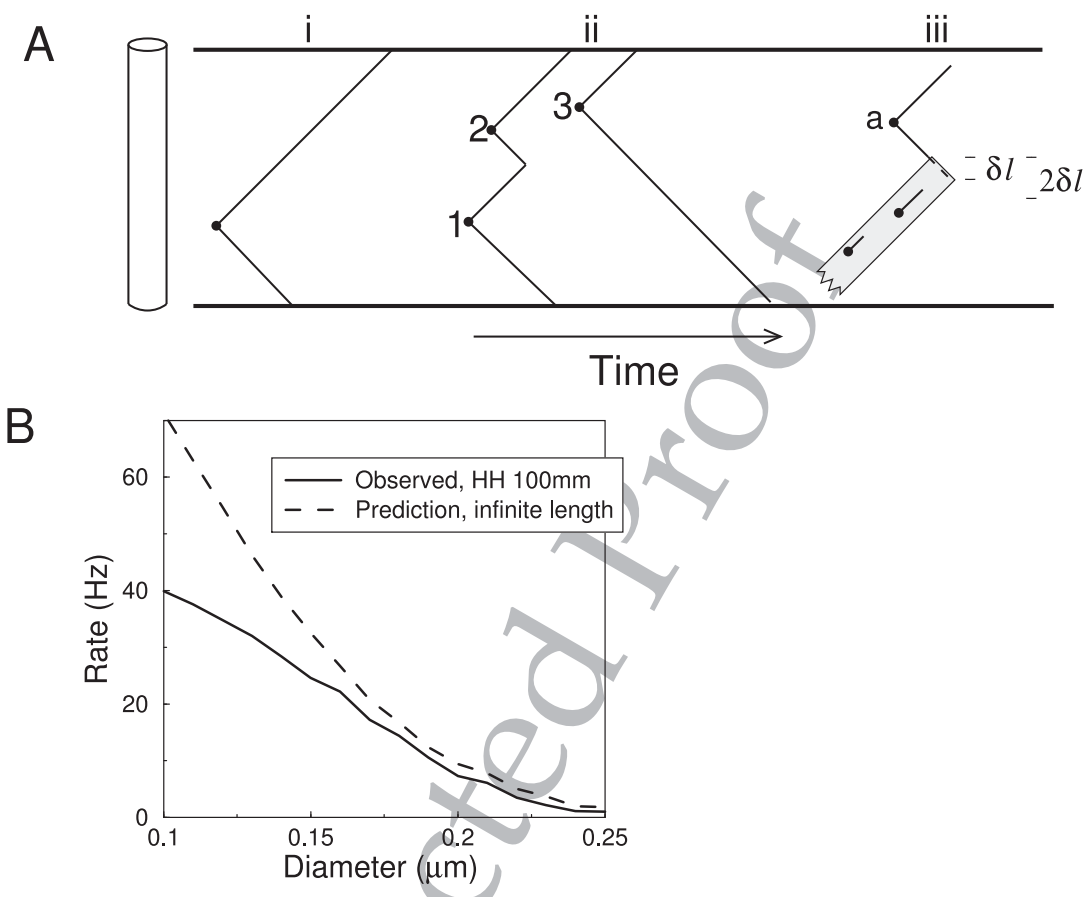

Figure 3: (A) Cartoon of (i) unhindered propagation of a single spontaneous spike, (ii) illustration of two spikes colliding (the third spike does not collide), and (iii) diagram used for calculating the spontaneous rate in the limit of very long axons or high generation rates. (B) Predicted spontaneous rate for axon of infinite length (dashed line, equation 3.4) and the rate extracted from $\mathrm{HH}$ simulations with $100 \mathrm{~mm}$ length (solid line). We extracted the propagation speed $v$ and the generation rate $\rho_{m}$ from the simulation.

In Figure $2 \mathrm{~A}$ we plot these terms as extracted from the simulations. For the shortest axons, most spikes are generated at the ends. With longer axons, the rate of spikes generated anywhere else increases linearly with the length, but the collision rate also increases linearly.

Collisions become significant if it is likely that there are multiple spikes propagating simultaneously in the axon. This happens when $f \tau \approx 1$, where $\tau$ is the time for a spike to propagate from end to end $\tau=L / v$. With equation 2.2 , this gives the axon length for which collisions become important, $L_{\text {coll }}$, as

$$
L_{\text {coll }}=\frac{1}{\rho_{m}}\left(-r_{e}+\sqrt{r_{e}^{2}+\rho_{m} v}\right) .
$$


This is plotted in Figure 2B. For the HH system, this length is always larger than the length for which spikes generated in the rest of the axon are dominant. Thus, when collisions start to appear, the spontaneous rate is dominated by the spikes generated in the rest of the axon, and so $L_{\text {coll }} \approx$ $\sqrt{v / \rho_{m}}$. As explored in detail below, when $L \gg L_{\text {coll }}$, the firing rate becomes independent of the axon length, as can be seen in Figures 1A and 2A.

In summary, there are four regimes in the $\mathrm{HH}$ axon that explain the shape of the graph of spontaneous rate versus axon length (see Figure 1A):

1. $L \ll \Lambda$ : The spontaneous spikes are mainly generated at the ends with a rate that decreases with length; no collisions occur.

2. $\Lambda \ll L \ll L_{c}$ : The spontaneous spikes are mainly generated at the ends. No collisions occur, and the rate is roughly constant.

3. $L_{c} \ll L \ll L_{\text {coll }}$ : The spontaneous spikes are mainly generated in the rest of the axon, no collisions occur, and the spontaneous rate increases linearly with length.

4. $L \gg L_{\text {coll }}$ : The spontaneous spikes are mainly generated in the rest of the axon, most spikes collide, and the rate is independent of length.

This is an idealized description that is meant to aid understanding; in practice, the regimes will overlap. Changes in the ion channel properties and densities as well as the geometry can further alter the classification and ordering of regimes. For instance, if the propagation speed is slower, collisions will be more likely ( $L_{\text {coll }}$ decreases). Or, as another example, if one axon end has a soma attached to it, or it receives strong inhibition, no spontaneous spikes are expected to originate there. In contrast, local depolarization can increase rates.

\section{Simplified Models}

In order to better understand the effect of collisions in long axons, we develop an abstract model of the action potential generation and propagation. Propagation failure is assumed to be negligible; indeed, we never observed it in our Hodgkin-Huxley simulations. Spike generation probability is a function of the position along the axon. A simple model is to have a rate $r_{e}$ at both ends and a rate per length $\rho_{m}$ along the rest of the axon; that is, the Poisson rate density is $\rho(x)=[\delta(x)+\delta(x-L)] r_{e}+\rho_{m}$. This system is simulated with an cellular automaton using a discretized axon, using three rules: it (1) creates spikes according to a Poisson process according to density $\rho(x),(2)$ propagates the generated spikes toward left and right from the initiation point, and (3) annihilates colliding spikes.

3.1 Generators on Axon Ends Only. We first analyze the idealized case when spikes are generated at the ends only. We assume that the axon length is long enough so that $r_{e}$ is constant. As above, we define the propagation time $\tau=L / v$ as the time it takes to travel from one end of the axon to the 
other. The only dimensionless parameter is $r_{e} \tau$. We find (see the appendix) that due to collisions, the observed rate becomes

$$
f_{o b s}=r_{e}\left(1+\frac{1}{1+2 r_{e} \tau}\right) \text {. }
$$

For low rates, collisions should not occur; hence, $f_{o b s}=2 r_{e}$. However, at high rates and long $\tau$, every spike generated on one end will annihilate with one from the other end; hence, $f_{o b s}=r_{e}$. The characteristic length for the switchover is $v / r_{e}$.

3.2 Uniform Generation Along the Axon. Next, we assume constant and uniform generation along the axon (a so-called Poisson sheet). In the absence of collisions, the spontaneous rate obeys $f_{\text {obs }}=\rho_{m} L$ (see equation 2.2). The first correction will be the occasional collision of two spikes (see Figure 3Aii). The probability for such events is proportional to the rate squared. It can be calculated exactly (see the appendix):

$$
f_{o b s}=\rho_{m} L\left[1-\frac{1}{3} \rho_{m} L^{2} / v+\mathrm{O}\left(\rho_{m}^{2}\right)\right] \text {. }
$$

While in principle one can try to systematically expand to include higherorder corrections, this quickly becomes unwieldy. Figure 3Aii illustrates why this calculation is hard: if the top left spike (number 2 in Figure 3) had not occurred, the top-right spike (number 3) would have collided with the bottom-left spike (number 1). In other words, the collision pattern can depend on the full spike history.

But while a general expression of spontaneous rate in terms of $\rho_{m}$ seems out of reach, the output rate becomes independent of the length of the axon in the limit of long axons $\left(L \gg L_{\text {coll }}\right)$. This can seen as follows. When the rates are high, most spikes will not propagate along the full axon; instead, they will collide. Apart from the ends, the axon behaves uniformly. Because the probability of collision is approximately uniform and constant, the spike length $l$ (i.e., the distance that a spike travels from initiation to collision point) will be distributed exponentially,

$$
P(l)=\frac{1}{\lambda} \exp (-l / \lambda)
$$

where $\lambda$ is the mean distance a spike travels before it collides.

Next, we wish to know the dependence of this mean distance on the generation rate. It follows from a self-consistency argument. Consider the spike labeled a in Figure 3Aiii. The collision process can be seen as a hazard process, where the hazard of a collision is given by the presence of other spikes. The hazard probability $h$ is the probability of the presence of another 
spike in infinitesimal length $\delta l$. Consider the shaded strip in Figure 3Aiii. In the limit of small $\delta l$, the colliding spikes present in this strip can be described by a binary random process with an on-rate that equals the generation rate, $2 \rho_{m} \delta l$, where the factor 2 is a geometrical factor (see Figure 3Aiii). The offrate of the process is the reciprocal of the mean time between collisions $v / \lambda$. As required by self-consistency, this process yields colliding spikes with a length that is exponentially distributed and mean length $\lambda$.

The hazard is thus $h=\frac{2 \rho_{m} \delta l}{2 \rho_{m} \delta l+v / \lambda} \approx 2 \rho_{m} \lambda \delta l / v$. This hazard will reduce the survival probability of the original spike according to $P(l+\delta l)=P(l)-$ $h P(l)$, so that $P(l) \propto \exp (-l h / \delta l)$. Identification with equation 3.3, gives the $2 \rho_{m} \lambda / v=1 / \lambda$, from which the mean spike length as a function of initiation rate follows as $\lambda=\sqrt{v / 2 \rho_{m}}$.

The final step is to compute the output rate from the mean spike length. Imagine that one observes the spike rate at a given location in the axon. The average number of observed spikes will be the product of the total number of initiations $\left(\rho_{m} L\right)$, the probability of having propagated in that location $(\lambda / L)$, and finally a factor 2 because every initiation leads to two spikes traveling in opposite direction. Thus, we have

$$
f_{o b s}=2 \lambda \rho_{m}=\sqrt{2 v \rho_{m}} \text {. }
$$

This matches the simulations of the reduced model (not shown) but also the full $\mathrm{HH}$ model well (see Figure 3B). Note that because $L_{\text {coll }} \gg L_{c}$ for the $\mathrm{HH}$ axon, the uniform generation assumption is valid. Perfect fits should not be expected because for very narrow axons, refractory effects will limit the rate in the HH model, while for very wide axons, the length would need to be even longer than $100 \mathrm{~mm}$ to ensure that we are in the collision-dominated regime.

3.3 Variability of Spontaneous Spikes. In single compartments and axons much shorter than $L_{\text {coll }}$, the spontaneous spikes in the reduced model will follow Poisson statistics, and thus the interspike intervals have an exponential interval distribution. However, in longer axons, the spontaneous spikês have more regular statistics. The intuition is that when by chance at some time many spikes are generated, the collision rate will increase as well, thus smoothing out some of the variability in the rate. To study this separately from the $\mathrm{HH}$ dynamics, including refractoriness and mainly for reasons of efficiency, we use the simplified Poisson sheet model, with a length $L=10 L_{\text {coll }}$ with a spontaneous rate of about $10 \mathrm{~Hz}$, assuming that spikes are generated uniformly throughout the axon $\left(\rho(x)=\rho_{m}\right)$.

Due to collisions, the interspike interval distribution is more regular than a Poisson process (see Figure 4, top left panel). This can be further analyzed by sorting the spikes according to their direction of travel. In 


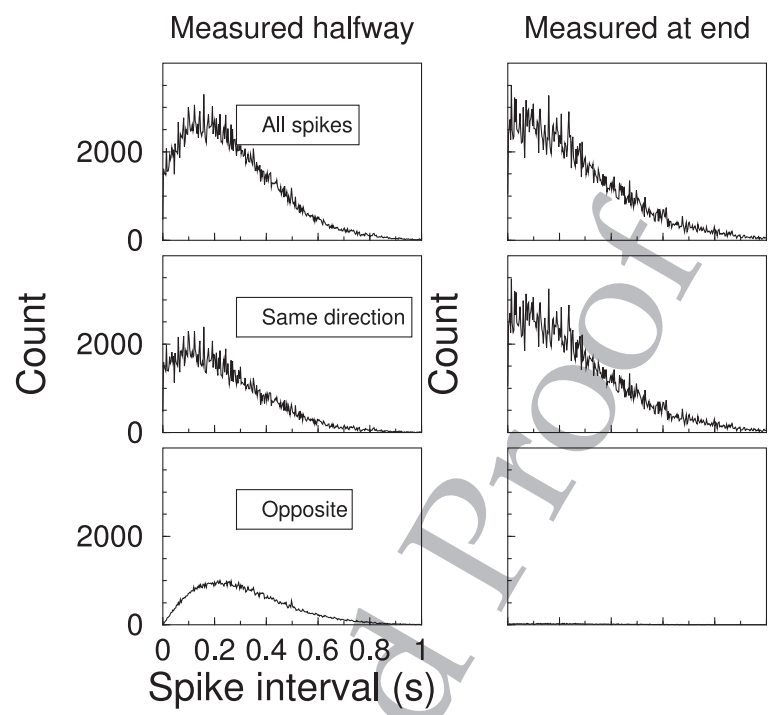

Figure 4: Interspike time interval histograms in an axon with length $10 \mathrm{~L}_{\text {coll }}$. (Left) Top: Counting all intervals. Middle: events where the subsequent spikes travel in the same direction. Bottom: events where the subsequent spikes travel in opposite direction. The variability is sub-Poisson $\left(C V_{i s i} \approx 0.67\right)$ in the middle of the axon (left column), but more Poisson-like at the end of the axon $\left(\mathrm{CV}_{\text {isi }} \approx\right.$ 0.78 , right column). This is due to the fact that most spikes there travel in the same direction (toward the end). Virtually no events have alternating directions at the end of the axon (bottom right).

particular, while the probability for a leftward-going spike to be followed by another leftward-going spike approximately resembles a Poisson process, a leftward-going spike is unlikely to be immediately followed by a rightward-traveling spike, as the second spike is likely to collide with a recent spike traveling in the opposite direction. As a result, the probability for intervals where the spikes travel in opposite directions is small for short intervals (bottom left panel, numerically resembling a gamma distribution with shape parameter $k=2$ ). When measuring the interval statistics irrespective of the propagation directions, the mix of these two processes leads to a process of sub-Poisson variability with an interspike interval coefficient of variation $(\mathrm{CV})$ of about 0.67 . While away from the ends, the rates of left-going and right-going spikes are equal; near the ends of the axon, only a few spontaneous spikes travel toward the middle. As a result, the interval distribution is dominated by spikes traveling in the same direction and is more Poisson-like near the ends and has a higher CV (see Figure 4, 
right panels). We also examined the effect of axon length. The CV does not drop further for longer axons, demonstrating that the system is well approximated by the collision-dominated regime. For shorter axons, the CV approaches one (more Poisson-like).

3.4 Effect on Signal Transmission. Finally, still using the Poisson-sheet model, we consider a situation where the neuron tries to send informative spikes down the axon. We analyze the spikes arriving at the other end of the axon where one could image a synaptic terminal to be present. We consider the transmission of a short burst of spikes through the axon and measure whether the spikes arrive at the terminal at the expected time (see Figure 5A). As one can observe, the first spike has only a limited probability of being transmitted, while later spikes are more likely to be transmitted (see Figure 5B). The reason is that the first spike has a high probability of encountering a spontaneously generated spike and thus being annihilated. The first spikes act as a snowplow, clearing the way for the subsequent spikes.

The probability that a colliding spike is present is proportional to the area of the striped triangle in Figure $4 C$, which equals $\rho_{m} L \tau=\left(L / L_{\text {coll }}\right)^{2}$. Under a Poisson assumption, the probability for transmission without collision equals exp $\left[-\left(L / L_{\text {coll }}\right)^{2}\right]$. For a spike much later in the train, the collision probability is equal to the slice $P=\rho_{m} L t_{i s i}$, where $t_{i s i}$ is the time between the spikes in the train, which is much smaller (hatched region in Figure 4B). The transmission probabilities for Figure $4 \mathrm{~B}$ are accordingly 0.37 for the first spike and 0.8 for the later spikes (for the $50 \mathrm{~Hz}$ train), which matches the simulations reasonably well. The higher the frequency of the train, the less probable it is that a spontaneous spike occurs between the spikes; hence, the reliability increases with the frequency of the train (see Figure 4B). This is different from most other types of noise in spiking, as usually the first spike is the most reliable, while subsequent ones are either jittered or unreliable (Mainen \& Sejnowski, 1995; van Rossum, O'Brien, \& Smith, 2003).

In addition to the spike times of the arriving train, one can study the total spike count. We measure the output rate as a function of the driving rate for a long train. The spontaneous activity will cause output spikes in the absence of somatically driven spikes (the abscissa in Figure 5D). However, unlike a single-compartment model, due to collisions, the output rate is not simply the sum of driven and spontaneous spikes. Instead, the driven spikes partly collide with the spontaneous spikes. As a result of this replacement noise, the resulting rate resembles the smoothed maximum of spontaneous and driven rates (see Figure $4 \mathrm{D}$, thick solid curve). For comparison, the mathematical maximum of spontaneous and driven rates is shown by the thin line. Due to the replacement noise, small stimuli will hardly increase the output rate, and the signal-to-noise ratio for such stimuli will be particularly poor. 
A
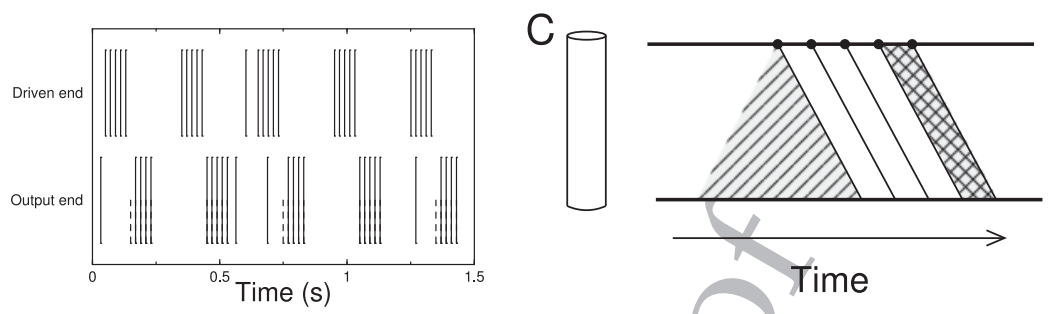

$\mathrm{B}$

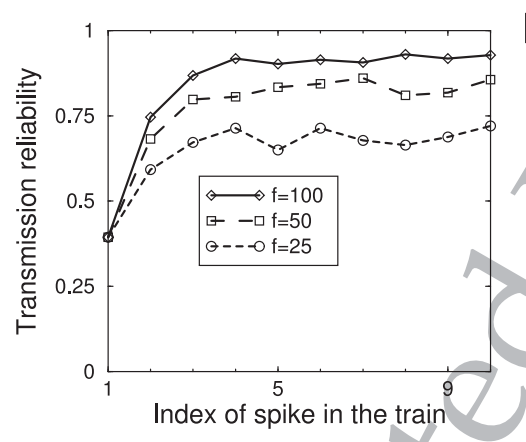

D

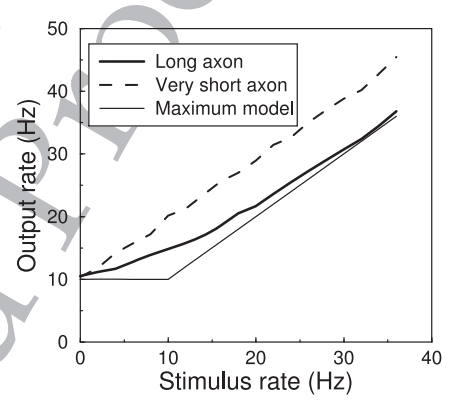

Figure 5: (A) Raster plot of a short simulation where the spike trains are generated at one end of the axon (top) and received at the other end of the axon (bottom). The short dashed bars in the lower row indicate when spikes would arrive had they not collided. In particular, the first spikes in the train are vulnerable to transmission failure. Some spontaneous spikes can be observed at either end. (B) The transmission probability of a spike from one end of the axon to the other for various stimulus frequencies. Due to collisions, the first spike has a low transmission probability, but later ones encounter only few spontaneous spikes on their way and have a high transmission probability, in particular at high stimulus frequencies. (C) Diagram to calculate the transmission probabilities. The first (left-most) spike in the train has a high collision probability (shaded triangle region), but later spikes have a lower one (hashed region). (D) The/output rate as a function of stimulus rate in a long axon (thick solid curve). Low-stimulus frequencies barely increase the output rate. In contrast, in a single-compartment model with spontaneous Poisson spikes, the output rate is simply the sum of spontaneous and driven rates (dashed line). Axon of length $L=L_{\text {coll }}, 10 \mathrm{~Hz}$ spontaneous rate.

\section{Discussion}

Some effects of ion channel noise have previously been studied in dendrites and axons. For instance, as the spike propagates, the speed varies, resulting in jitter of the spike arrival times at the axon terminals (Verveen \& Derksen, 1968; Lass \& Abeles, 1975; Horikawa, 1991; Faisal \& Laughlin, 2007). We 
have considered spontaneous spike generation in axons. In thin axons, the spontaneous rates can be substantial. The spontaneous spikes are preferably generated at the ends of the axons, with a much lower rate in the rest of the axon. However, for long axons $\left(L \gg L_{c}\right)$, the contribution of the rest of the axon dominates. As a result, we find a nonmonotonic relation between the spontaneous rate and the length of the axon. The minimal firing rate occurs at a length of about $1 \mathrm{~mm}$, which accidentally coincides with the length used in Faisal et al. (2005). Therefore, our results put even more stringent bounds on the minimal diameter to prevent high spontaneous rates than suggested there. In particular, long axons might need to be somewhat thicker.

For even longer axons $\left(L \gg L_{\text {coll }}\right)$, we find that the spontaneous rate saturates as spikes start to collide with each other. Our theory predicts when this will happen and what the expected/spike rate will be. Many questions are left about the collision regime, even in the simplified model. What is the firing rate for an intermediate-length axon, between equation 3.2 and equation 3.4? What if refractory time is included? And what is the exact interval distribution for such axons?

The most prominent question is whether spontaneous spikes caused by stochastic processes occur in biology and at what rate. If there is strong evolutionary pressure to reduce wiring diameter, as has been suggested (Faisal et al., 2005), it is not unlikely that the nervous system operates in a regime where some spontaneous spikes occur.

Related to that question is our choice of model system, namely the standard Hodgkin-Huxley model. The simulation presented in Faisal et al. (2005) shows that the spontaneous rates in $\mathrm{HH}$ and axon models tuned to mammalian fibers are comparable. Nevertheless, the exact characterization of the voltage-gated channels and their densities in long axons required to make quantitative predictions is far from complete and the spontaneous rate's exponential dependence on the fluctuations (see equation 2.1) makes it sensitive to precise model details. Whether spontaneous spikes are mainly generated throughout the axon or at the ends is similarly difficult to determine. In this light, our study should be seen as a demarcation of the various possible regimes. However, our results on the interval statistics and transmission might be used to test whether axons are in the collision regime. The conduction speed in mammalian C-fibers is on the order of $1 \mathrm{~m} / \mathrm{s}$ (compare to $0.2 \mathrm{~m} / \mathrm{s}$ for the $\mathrm{HH}$ model), so that for spontaneous rates of $1 \mathrm{~Hz}$, the collision length is $L_{\text {coll }} \approx 1 \mathrm{~m}$.

\section{Appendix: Analytical Approach}

A.1 Spontaneous Rate for Generation at Ends Only. To calculate the spontaneous rate when spikes are generated at the ends of the axon only, we use the following approach. We assume that during a time $T$, there is a total of $n$ spikes generated, of which $m$ are on one end of the axon and $n-m$ at the other side. Both $m$ and $n-m$ are independently Poisson distributed. In 


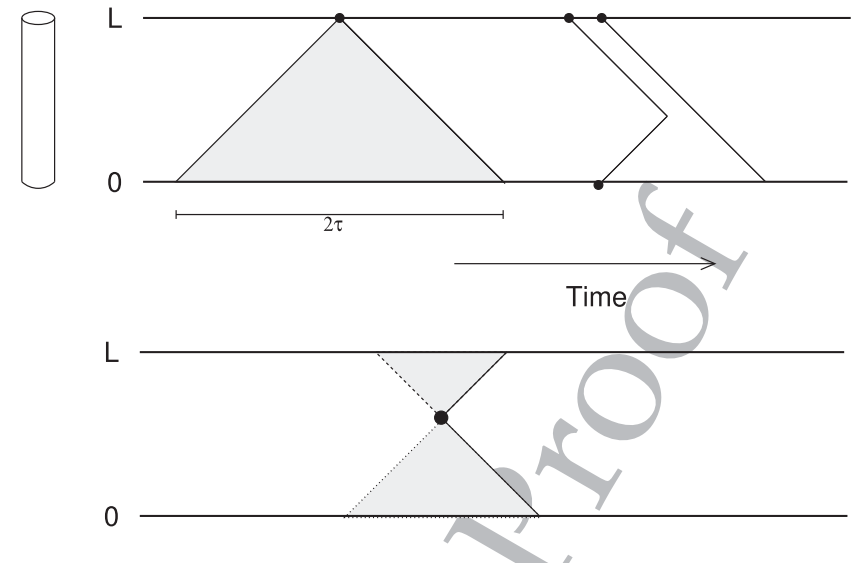

Figure 6: (Top) When spikes are generated at the ends only, a second spike generated at the bottom on the indicated interval $2 \tau$ will collide with the top spike. Right: Note that if the first top spike has collided, a second top spike propagates freely. (Bottom) When spikes are generated throughout the axon, a second spike will collide with the indicated one if it falls anywhere in the shaded triangle region.

this case, each spike can at most collide with one other spike. We calculate the probability that a given spike at the top end collides with any of the $n-m$ spikes from the other end. The probability for a given top-end spike to collide with a bottom-end spike is $2 \tau / T$ (see Figure 6(top)). Because the events are independent, the probability for colliding with any spike is $p_{0}=1-(1-2 \tau / T)^{n-m}$. We ignore effects at the start and end of the time, which is allowed as we will take the limit $T \rightarrow \infty$.

When a collision happens, the collided spikes should be removed from the pool and the subsequent collision probability changes. For instance, if $m=2$, there are two possible ways that just one collision takes place: (1) the first spike does not collide (probability $q_{0} \equiv 1-p_{0}$ ) and the second does (probability $p_{0}$ ), and (2) the first spike collides $p_{0}$ and the second does not. However, in the second case, we have to update the collision probability as there is one fewer spike to collide with. The probability for the second spike not colliding becomes $q_{1}=1-p_{1}$, where $p_{i}=1-(1-2 \tau / T)^{n-m-i} \approx$ $1-\exp [-2(n-m-i) \tau / T]$ is the probability for collision after $i$ spikes have collided already and have thus been removed.

The probability for $k$ collisions in time $T$ given $m$ spikes originating from one end and $n$ from the other end is

$$
p(k \mid m, n)=\left(\prod_{l=0}^{k-1} p_{l}\right) \sum_{\substack{i_{o}, i_{1} \ldots i_{k}=0 \\ \sum_{l} i_{l}=m-k}}^{m-k}\left[\prod_{l=0}^{k} q_{l}^{i_{l}}\right] .
$$


The term in the parentheses corresponds to the probability of $k$ spikes colliding, while the sum term corresponds to all configurations of other spikes not colliding. The sum runs from 0 to $m-k$ for each index $i$ but is restricted so that the sum of indices $\sum_{l} i_{l}=m-k$. This ensures that there are exactly $m-k$ spikes that do not collide. The average observed rate follows as $f_{o b s}=(n-\langle k\rangle) / T$.

Equation A.1 is a polynomial in $\tau$ that quickly grows in complexity with increasing $k$ and $m$. Despite having an explicit expression for $p(k)$, a systematic expansion for $\langle k\rangle$ in the limit of large $n, m, T$ is hard. Using Mathematica, we numerically expanded the mean $k$ with regard to $\tau / T$ and find that the terms behave as $\langle k\rangle=m\left[2 m \tau / T-4(m \tau / T)^{2}+\ldots\right]$, so that the number of observed spikes is $n-\langle k\rangle \approx 2 m-2 m(m \tau / T)+4 m(m \tau / T)^{2}+\ldots$ matching the relation found above (see equation 3.1).

A.2 Perturbation Expansion for Spontaneous Rate for Uniform Generation. Here we derive equation 3.2 , the collision rate in the first order in the limit of low rates assuming a constant, uniform generation throughout the axon with a rate $\rho_{m}$, although the formalism in principle can be extended to varying rates. The mean number of spikes generated during a time $T$ is $n=\rho_{m} L T$, so the observed rate will be $\rho_{m} L$ if there are no collisions.

The first correction corresponds to two spikes colliding so that only one spike remains. Consider a first spike generated somewhere, sometime in the axon (see Figure 6(bottom)). If the second spike is generated in either shaded triangular region, it will always collide with the first spike. Given a spike generated at a time $t_{1}$ and location $x_{1}$, the probability of a collision with the second spike $\left(x_{2}, t_{2}\right)$ is given by the integral over these shaded regions divided by the total area, $L T$. This can be formally expressed using integrals with the Heaviside function $H()$ as

$$
\begin{aligned}
& p\left(x_{1}, t_{1}\right) \\
& =\frac{1}{L T} \int_{0}^{x_{1}} d x_{2} \int_{0}^{T} d t_{2} H\left(x_{1} / v+t_{1}-x_{2} / v-t_{2}\right) H\left(x_{1} / v-t_{1}-x_{2} / v+t_{2}\right) \\
& +\int_{x_{1}}^{L} d x_{2} \int_{0}^{T} d t_{2} H\left(-x_{1} / v-t_{1}+x_{2} / v+t_{2}\right) H\left(-x_{1} / v+t_{1}+x_{2} / v-t_{2}\right) .
\end{aligned}
$$

Averaged over all possible positions of the first spike and in the limit of $T \rightarrow \infty$,

$$
\begin{aligned}
p & =\frac{1}{L T} \int_{0}^{L} d x_{1} \int_{0}^{T} d t_{1} p\left(x_{1}, t_{1}\right) \\
& =\frac{2}{3} \frac{\tau}{T} .
\end{aligned}
$$


This is the collision probability per spike pair. Because there are $n^{2} / 2$ pairs, the collision rate is $f_{\text {coll }}=n^{2} \tau / 3=\rho_{m}^{2} L^{3} T / 3 v$, which leads directly to equation 3.2.

The same arguments can be followed for the possible collisions with three spikes. Obviously two spikes can collide, while a third one propagates independently (order $\rho_{m}^{2}$ ). However, the interaction of three spikes (order $\rho_{m}^{3}$ ) can lead to either one or two collisions, depending on the precise configuration of the initiation points. These effects make higher-order expansion complicated and cumbersome.

\section{Acknowledgments}

Discussions with Sue Fleetwood-Walker, Jean-Marc Luck, and Eleni Vasilaki are gratefully acknowledged. C.O.D. was supported by EPSRC/BBSRC/ MRC through the Doctoral Training Centre in Neuroinformatics.

\section{References}

Cannon, R. C., O’Donnell, C., \& Nolan, M. F. (2010). Stochastic ion channel gating in dendritic neurons: Morphology dependence and probabilistic synaptic activation of dendritic spikes. PLoS Computational Biology, 6(8), e1000886.

Carnevale, N. T., \& Hines, M. L. (2006). The neuron book. Cambridge: Cambridge University Press.

Chow, C. C., \& White, J. A. (1996). Spontaneous action potentials due to channel fluctuations. Biophys. J., 71, 3013-3021.

Faisal, A. A., \& Laughlin, S. B. (2007). Stochastic simulations on the reliability of action potential propagation in thin axons. PLoS Computational Biology, 3(5), e79.

Faisal, A. A., White, J. A., \& Laughlin, S. B. (2005). Ion-channel noise places limits on the miniaturization of the brain's wiring. Current Biology, 15(12), 11431149.

Horikawa, Y. (1991). Noise effects on spike propagation in the stochastic HodgkinHuxley models. Biological Cybernetics, 66(1), 19-25.

Keener, J. P., \& Newby, J. M. (2011). Perturbation analysis of spontaneous action potential initiation by stochastic ion channels. Phys. Rev. E, Stat. Nonlin. Soft. Matter Phys., 84(1 Pt. 1), 011918.

Lass, Y., \& Abeles, M. (1975). Transmission of information by the axon. I. Noise and memory in the myelinated nerve fiber of the frog. Biol. Cybern., 19(2), 61-67.

Mainen, Z. F., \& Sejnowski, T. J. (1995). Reliability of spike timing in neocortical neurons. Science, 268, 1503-1506.

Newby, J. M., Bressloff, P. C., \& Keener, J. P. (2013). Breakdown of fast-slow analysis in an excitable system with channel noise. Phys. Rev. Lett., 111(12), 128101.

O'Donnell, C., \& Van Rossum, M. C. (2014). Systematic analysis of the contributions of stochastic voltage gated channels to neuronal noise. Frontiers in Computational Neuroscience, 8, 105.

Tuckwell, H. C., \& Walsh, J. B. (1983). Random currents through nerve membranes. Biol. Cybern., 49, 99-110. 
van Rossum, M.C.W., O’Brien, B. J., \& Smith, R. G. (2003). The effects of noise on the timing precision of retinal ganglion cells. J. Neurophysiol., 89, 2406-2419.

Verveen, A., \& Derksen, H. (1968). Fluctuation phenomena in nerve membrane. Proceedings of the IEEE, 56(6), 906-916.

Received August 13, 2014; accepted September 29, 2014. 